\title{
CONTRIBUTIONS TO THE THEORY OF THE LARGEST CLAIM COVER
}

\author{
J. KUPPER \\ Zurich
}

In the wake of the technical development of our era we are increasingly faced with claims of extremely large amounts.

Whereas even in the past century large claims were mostly due to elemental or natural forces such as earthquakes, hurricanes and floods, there are today other causes, conditioned by human factors, that have considerably increased in importance. Another fact to be observed is that the risks that are passed on to the insurance market assume a more serious character year by year. Large building complexes, giant tankers, containers, atomic power stations, dams and jumbo jets pose for the insurers problems which are in no way easy to solve, since the risk covers required touch the limits of market capacity and their rating is subject to great uncertainties. At the same time, the readiness to underwrite such covers is a matter of utmost importance for the private insurance industry.

For the coverage of such large risks, various insurance forms have been developed in the past years within the range of non-proportional methods. Besides the excess of loss and stop loss covers in their usual form, the cumulative loss cover and the largest claim cover have attracted particular attention. The cumulative risk cover has, for example, been dealt with in [II] $\left.{ }^{1}\right)$ and has also been frequently offered in practice. The coverage of the largest claim or, more generally, of the sum of the $n$ largest claims does not appear to have gained a proper foothold in practice in spite of various valuable contributions in this field-considering particularly [2], [3], [5], [8] and [I4]. The fact that it was during $1963 / 64$ that various authors analysed these problems is no coincidence, for it was then that in two ASTIN Colloquia these topics were discussed.

The purpose of this presentation is to take up once again these

1) Figures in [ ] refer to the list of references at the end of this paper. 
thoughts and to give some further results in continuation of the papers by Ammeter. The author wishes to take this opportunity of thanking Dr. Ammeter for pointing out to him this interesting line of subjects.

\section{Basic formulas of the largest claim distribution}

The risk process in non-life insurance is characterised by two stochastic variables, the number of claims and the amount of claims.

If

$P_{r}(t)$ represents the probability that exactly $r$ claims occur in the period observed, with the expected number of claims $t$,

$S(x)$ represents the probability that upon occurence of a claim its amount is $\leq x$, and

$S^{* r}(x)$ represents the $r^{\text {th }}$ convolution of the distribution function $S(x)$,

then, the total loss can be expressed on certain simplifying assumptions by the well-known formula

$$
F(x, t)=\sum_{r-0}^{\infty} P_{r}(t) S^{*}(x)
$$

Further, for the first two moments one finds (the subscript conforms to the corresponding distribution)

$$
\begin{aligned}
& \mu_{F}=\mu_{P} \mu_{S} \\
& \sigma_{F}^{2}=\mu_{P} \sigma_{S}^{2}+\sigma_{P}^{2} \mu_{S}^{2}
\end{aligned}
$$

Franckx has shown in [8] how the distribution of the largest claim can be based very simply on the two fundamental distributions of the risk process. In particular, if

$$
Q(s, t)=\sum_{r=0}^{\infty} P_{r}(t) s^{r}
$$

is the generating function of the distribution of the number of claims, we have for the distribution of the largest claim $m$ of a portfolio the relationship

$$
H(m, t)=Q[S(m), t]
$$


By differentiating with respect to $m$, one obtains the density function $h(m, t)$ and from this the moments of the distribution can be determined. For example, the mean is

$$
\mu_{H}=\int_{0}^{\infty} m h(m, t) d m
$$

For the distribution of the numter of claims, the classical model assumes a Poisson process. The assumption of a negative binomial distribution has, however, proved to be more flexible. Let this be of the form

$$
P_{r}(t)=\left(\begin{array}{c}
T+r-\mathbf{I} \\
r
\end{array}\right)\left(\frac{T}{T+t}\right)^{T}\left(\frac{t}{T+t}\right)^{r}
$$

with the given fluctuation parameter $T$. As the generating function in this case we have

$$
Q(s, t)=\left[\mathrm{I}+(\mathrm{I}-s) \frac{t}{T}\right]^{-T}
$$

so that for the distribution and the density function of the largest claim the resultant expressions are

$$
\begin{aligned}
& H(m, t)=\left\{\mathrm{I}+[\mathrm{I}-S(m)] \frac{t}{T}\right\}^{-T} \\
& h(m, t)=t s(m)\left\{\mathrm{I}+[\mathrm{I}-S(m)] \frac{t}{T}\right\}^{-T-1}
\end{aligned}
$$

$s(m)$ is the density function of claim amounts.

\section{Variations in the basic distributions}

If in (6) $T \rightarrow \infty$, then the classical Poisson distribution results with the generating function

$$
Q(s, t)=e^{-(1-8) t}
$$

In [2] and [3] Ammeter has closely investigated only this case and has assumed moreover that the distribution of the amount of claims obeys a Pareto law

$$
S(m)=\mathrm{I}-m^{1-\alpha}
$$

The choice of this special form is not only accounted for by the fact that the results produced can be expressed in quite an elegant 
way but it appears that especially for large claims the adjustment through a Pareto distribution often turns out to be amazingly good. The investigations of Benktander and Segerdahl ([6] and [7]) have moreover furnished additional grounds for the assumption made by Ammeter.

The Pareto distribution defined under (Io) exists in the interval $(I, \infty)$ and has the mean

$$
\mu_{S P}=\frac{\alpha-\mathrm{I}}{\alpha-2}(\alpha>2)
$$

The $k^{\text {th }}$ moment of the distribution is only then finite when $\alpha>k+\mathrm{I}$. However, this restriction is not a very serious one in practice and it can be overcome by a suitable truncation of the distribution.

Considering the Poisson Case (9) the formula (5) reads

$$
\mu_{H}=t \int_{0}^{\infty} m s(m) e^{-t[1-S(m)]} d m
$$

If one uses the distribution (I0) here, then one obtains, after some transformations, the result already found by Ammeter

$$
\mu_{H P}=t^{1 /(\alpha-1)} \Gamma_{t}\left(\frac{\mathrm{I}}{\mu_{S P}}\right)
$$

where $\Gamma_{t}$ signifies the incomplete Gamma function. For practical purposes one can, in most cases, compute with $\Gamma$ itself instead of with $\Gamma_{t}$.

However, the most usual assumption for the distribution of claim amounts-on account of the relatively complicated evaluation of formula (I) - is perhaps an exponential distribution. Various authors (for example [I], [9] and [I3]) have already made use of this possibility which is admittedly fairly crude. It might, therefore, be of interest to elucidate somewhat the effects of the application of such a distribution instead of (IO).

Let the exponential distribution be so chosen that the range and the mean remain unchanged as compared with (IO). This delivers the formula

$$
S(m)=\mathrm{I}-e^{-(\alpha-2)(m-1)}
$$


with

$$
\mu_{S E}=\mathrm{I}+\frac{\mathrm{I}}{\alpha-2}=\frac{\alpha-\mathrm{I}}{\alpha-2}=\mu_{S P}
$$

In contrast to the Pareto distribution, there exist here, provided $\alpha>2$, all moments.

If with (I4) or its derivation one considers formula (I2) and further substitutes $u=t e^{-(\alpha-2)(m-1)}$, one obtains

$$
\begin{aligned}
\mu_{H E} & =\int_{0}^{t}\left(\mathrm{I}+\frac{\mathrm{I}}{\alpha-2} \log \frac{t}{u}\right) e^{-u} d u \\
& =\left(\mathrm{I}+\frac{\mathrm{I}}{\alpha-2} \log t\right)\left(\mathrm{I}-e^{-t}\right)-\frac{\mathrm{I}}{\alpha-2} \int_{0}^{t} e^{-u} \log u d u
\end{aligned}
$$

It can be shown that

$$
\int_{0}^{t} e^{-u} \log u d u=-C-E i(-t)-e^{-t} \log t
$$

where $C=0,5772157 \ldots$, is the Euler's constant and $-E i(-t)=$ $\int_{i}^{\infty} e^{-u} / u d u$ denotes the exponential integral.

Accordingly, where $t$ is large, one can compute with the formula

$$
\mu_{H E}=\mathrm{I}+\frac{\mathrm{I}}{\alpha-2}(C+\log t)
$$

without any loss of accuracy.

The results according to (I3) and (I7) are indicated in the following table for various $\alpha$ on the assumption that $t=$ Ioo. The values

\begin{tabular}{|c|c|c|c|c|c|c|}
\hline$\alpha$ & $\begin{array}{l}\mu_{F} \\
(\mathrm{I})\end{array}$ & $\begin{array}{l}\mu_{H P} \\
(2)\end{array}$ & $\frac{\mu^{H P}}{\mu_{F}}$ in $\%$ & $\begin{array}{c}\mu_{H E} \\
(4)\end{array}$ & $\frac{\mu_{H P}}{\mu_{F}}$ in $\%$ & $\frac{\frac{\mu_{H P}}{\mu_{H \Delta}}}{(6)}$ \\
\hline 2,25 & 500,00 & I 82,77 & 36,6 & $2 \mathrm{I}, 73$ & 4,3 & 8,4 \\
\hline 2,5 & 300,00 & 57,72 & 19,2 & 11,36 & 3,8 & 5,1 \\
\hline 2,75 & 233,33 & 28,73 & I 2,3 & $7,9 \mathrm{I}$ & 3,4 & 3,6 \\
\hline 3 & 200,00 & I 7,72 & 8,9 & 6,18 & 3,1 & 2,9 \\
\hline 3,5 & 166,67 & $9,4^{\circ}$ & 5,6 & 4,45 & 2,7 & $2, I$ \\
\hline 4 & I $5^{0,00}$ & 6,29 & 4,2 & 3,59 & 2,4 & $\mathrm{I}, 8$ \\
\hline 5 & I 33,33 & 3,87 & 2,9 & 2,73 & 2,0 & $\mathrm{I}, 4$ \\
\hline Io & I I $2,5^{\circ}$ & $\mathrm{I}, 80$ & I, 6 & 1,65 & I, 5 & $\mathrm{I}, \mathrm{I}$ \\
\hline$\infty$ & 100,00 & 1,00 & $I, O$ & $1, \infty$ & $\mathrm{I}, \mathrm{O}$ & $\mathrm{I}, \mathrm{O}$ \\
\hline
\end{tabular}
contained in the first three columns have been already given in [2].

TABLE I

Comparison of the means $\mu_{F}$ (total loss), $\mu_{H P}$ (the largest claim/Pareto) and $\mu_{H \mathrm{E}}$ (the largest claim/exponential) 
Since in the example chosen, $\frac{\text { IOo }}{\mu_{F}}=\frac{\mathrm{I}}{\mu_{S P}}=\frac{\mathrm{I}}{\mu_{S E}}$, the third and the fifth columns give an indication of the number of times by whichthe expected value of largest claim exceeds the mean of individual claims. It is clear that with increasing $\alpha$, i.e. with decreasing "danger" of the distribution $(\alpha=\infty$ signifies constant claim amounts) the given values must decrease. On the other hand, a comparison of the above two columns shows how significant the weight of the largest claim is for small $\alpha$ in the case of the Pareto distribution. For example, whereas for $\alpha=2,25$ and the exponential distribution as the distribution of claim amounts the largest claim constitutes only $4,3 \%$ of the total loss, in the case of the Pareto distribution it constitutes $36,6 \%$ !

This result suggests the conclusion that to operate with the exponential distribution would be hardly satisfactory in cases where large claims are likely. In the following studies, therefore, the Pareto distribution is always postulated.

Proceeding from formula (8) and using the distribution (Io), one obtains for the mean value (5) the expression

$$
\mu_{H}=t^{1 /(\alpha-1)} \int_{0}^{t} u^{1 /(\alpha-1)}\left(I+\frac{u}{T}\right)^{-T-1} d u
$$

after the usual substitution.

In deriving (13) it was assumed that $T \rightarrow \infty$. In the following, the case where $T=\mathrm{I}$ is examined. For $T=\mathrm{I}$, formula (6) reduces to

$$
P_{r}(t)=\frac{\mathrm{I}}{\mathrm{I}+t}\left(\frac{t}{\mathrm{I}+t}\right)^{r}
$$

in words, to a geometric distribution. As in the previous approximations, it may, without any great loss of accuracy, further be assumed that the upper limit of the integral can be taken to be $\infty$.

By using the method of partial fractions for the integrand and then integrating by parts one finds at first

$$
\int_{0}^{\infty} u^{1 /(\alpha-1)}(\mathrm{I}+u)^{-2} d u=\frac{\mathrm{I}}{\alpha-\mathrm{I}} \int_{0}^{\infty} u^{1 /(\alpha-1)}(\mathrm{I}+u)^{-1} d u
$$


But now, for $0<n<$ I

$$
\int_{0}^{\infty} u^{n-1}(\mathrm{I}+u)^{-1} d u=\frac{\pi}{\sin n \pi}=\Gamma(n) \Gamma(\mathrm{I}-n)
$$

holds good.

With (20) and (2I), one obtains for the expected value of the largest claim in the case of a geometric distribution

$$
\begin{aligned}
\mu_{H G} & =\frac{t^{1 /(\alpha-1)}}{\alpha-I} \Gamma\left(\frac{\alpha-2}{\alpha-I}\right) \Gamma\left(\frac{1}{\alpha-I}\right) \\
& =\Gamma\left(\frac{\alpha}{\alpha-I}\right) \mu_{H P}
\end{aligned}
$$

if $\mu_{H P}$ represents the approximate mean value ( $\Gamma$ instead of $\Gamma_{t}$ ) according to (I3).

For $2<\alpha<\infty$, the factor $\Gamma\left(\frac{\alpha}{\alpha-\mathrm{I}}\right)$ takes values in the interval (0.886, I), $\mu_{H G}$ therefore lies always slightly below $\mu_{H P}-\mathrm{a}$ result that could hardly be expected on intuition.

\section{Excess of loss and the largest claim cover}

Generally speaking, an insurance company would not undertake unlimited liability as has been assumed in the statements made so far; it will limit its liability to a maximum retention $M$. The effect of such an excess of loss reinsurance is that even the largest claim cannot exceed the amount $M$.

The probabilistic model can directly be adjusted through a "truncation" of the distribution of the largest claim. Actually this is not a truncation in the usual sense (transferring the residual mass beyond the point of truncation proportionately over the remaining interval) but a concentration (transferring the residual mass at the point of truncation $M$ ). All claims which turn out to be larger than $M$ are registered as $M$-claims (see in this connection [Io]).

Since in the case of Poisson and Pareto as basic distributions the formulas (8) change into

$$
\begin{aligned}
H(m, t) & =e^{-t m^{1-\alpha}} \\
h(m, t) & =t(\alpha-\mathrm{I}) m^{-\alpha} e^{-t m^{1-\alpha}}
\end{aligned}
$$


the new density of the distribution of the largest claim is

$$
\tilde{h}(m, t)=\left\{\begin{array}{lr}
h(m, t) & \mathrm{I} \leq m<M \\
\mathrm{I}-e^{-t M^{1-\alpha}} & m=M \\
0 & m>M
\end{array}\right.
$$

According to formula (5) we have, therefore, as the mean of this distribution

$$
\tilde{\mu}_{H}=t^{1 /(\alpha-1)}\left[\Gamma_{t}\left(\frac{\mathrm{I}}{\mu_{S P}}\right)-\Gamma_{M^{*}}\left(\frac{\mathrm{I}}{\mu_{S P}}\right)\right]+M\left(\mathrm{I}-e^{-M^{*}}\right)
$$

putting $M^{*}=t M^{1-\alpha}$ for abbreviation.

Obviously, for a fixed $M$ and large values of $t$, the expected largest claim $\tilde{\mu}_{H}$ tends to the retention $M$. This follows also from the above formula (25).

The influence of the concentration in (24) on the next largest claims can be investigated in a similar way. For example, the expression for the density function of the second largest claim without truncation (see [3]) is

$$
h^{(2)}(m, t)=t^{2}(\alpha-I) m^{1-2 \alpha} e^{-t m^{1-\alpha}}
$$

For the expected value of the second largest claim after concentration we get

$$
\tilde{\mu}_{H}^{(2)}=\int_{1}^{M} m h^{(2)}(m, t) d m+M\left(\mathrm{I}-e^{-M^{*}}-M^{*} e^{-M^{*}}\right)
$$

With the help of the recursion formula for the incomplete Gamma function

$$
\Gamma_{1}(x+\mathrm{I})=x \Gamma_{1}(x)-e^{-t} t^{x}
$$

and the result of $(25)^{1}$ ), we obtain for the integral in formula (27) the expression

$$
\begin{aligned}
\int_{1}^{M} m h^{(2)}(m, t) & d m= \\
= & \frac{\mathrm{I}}{\mu_{S P}}\left[\tilde{\mu}_{H}^{(1)}-M\left(\mathrm{I}-e^{-M^{*}}\right)\right]+t\left(-e^{-t}+e^{-M^{*} M^{2-\alpha}}\right)
\end{aligned}
$$

1) Analogically, we denote the expected value of the largest claim now by $\tilde{\mu}_{H^{(1)}}$. 
and thus, after a slight rearrangement,

$$
\tilde{\mu}_{H}^{(2)}=\frac{\mathrm{I}}{\mu_{S P}} \tilde{\mu}_{H}^{(1)}+\frac{M}{\alpha-\mathrm{I}}\left(\mathrm{I}-e^{-M^{*}}\right)-t e^{-t}
$$

The third term can generally be disregarded. For a fixed $M$ and large values of $t, \tilde{\mu}_{H}^{2}$. also tends to $M$ as can be expected intuitively.

Whereas for the untruncated largest claim distribution between the expected values of the largest and the second largest claim the relationship

$$
\mu_{H}^{(2)}=\frac{\mathrm{I}}{\mu_{S P}} \mu_{H}^{(1)}-t e^{-t}
$$

holds, an additional term must be taken into consideration for (28). If the term $t e^{-t}$ is once disregarded, the ratio of the expected values in formula (29) is independent of $t^{1}$ ), whereas in the case of a truncated distribution the ratio tends to $\mathrm{I}$ for increasing $t$.

\section{Influence of the largest claim upon a change in the retention}

In view of the position described in the introduction, the insurer will increasingly be compelled to take a more risk-taking attitude. The increase in his maximum retention will on the other hand affect the expected value of the largest claims. This influence, which may not be insignificant in considering a change, can be investigated with the help of the formula provided in section 3. As in that section, only the two largest claims are taken into account.

Let the new retention be $\hat{M}>M$, specifically $\hat{M}=2 M$.

Then

$$
\begin{aligned}
\hat{\tilde{\mu}}_{H}^{(1)}-\tilde{\mu}_{H}^{(1)}=t^{1 /(\alpha-1)}[ & {\left[{ }_{M^{*}}\left(\frac{\mathrm{I}}{\mu_{S P}}\right)-\Gamma_{\hat{M}^{*}}\left(\frac{\mathrm{I}}{\mu_{S P}}\right)\right]+} \\
& +\hat{M}\left(\mathrm{I}-e^{-\hat{M}^{*}}\right)-M\left(\mathrm{I}-e^{-M^{*}}\right)
\end{aligned}
$$

if, in a similar manner as before, $\hat{M}^{*}=t \hat{M}^{1-\alpha}$ is used.

1) This proposition generally holds good since the ratio of the expected values of the $n^{\text {th }}$ largest claim and of the largest claim can be approximately given by the simple formula

$$
\frac{\mu_{H}^{(n)}}{\mu_{H}^{(1)}}=\left(\frac{\mathrm{I}}{\mu_{S p}}+n-2\right)
$$


Also

$$
\begin{aligned}
\hat{\tilde{\mu}}_{H}^{(1)}+\hat{\tilde{\mu}}_{H}^{(2)}-\left(\tilde{\mu}_{H}^{(1)}+\tilde{\mu}_{H}^{(2)}\right) & =\left(\mathrm{I}+\frac{\mathrm{I}}{\mu_{S P}}\right)\left(\hat{\tilde{\mu}}_{H}^{(1)}-\tilde{\mu}_{H}^{(1)}\right)+ \\
+ & \left(\frac{\mathrm{I}}{\alpha-\mathrm{I}}\right)\left[\hat{M}\left(\mathrm{I}-e^{-\hat{M}^{*}}\right)-M\left(\mathrm{I}-e^{-M^{*}}\right)\right]
\end{aligned}
$$

or utilising (30)

$$
\begin{aligned}
&=\left(\mathrm{I}+\frac{\mathrm{I}}{\mu_{S P}}\right) t^{1 /(\alpha-1)} {\left[\Gamma_{M *}\left(\frac{\mathrm{I}}{\mu_{S P}}\right)-\Gamma_{\widehat{M}^{*}}\left(\frac{\mathrm{I}}{\mu_{S P}}\right)\right]+} \\
&+2\left[\hat{M}\left(\mathrm{I}-e^{-\hat{M}^{*}}\right)-M\left(\mathrm{I}-e^{-M^{*}}\right)\right]
\end{aligned}
$$

As can be readily seen, the increase represented by (3I) can be very easily derived from formula (30) by multiplying the first term of this relationship by the factor $\left(I+\frac{I}{\mu_{S P}}\right)=\frac{2 \alpha-3}{\alpha-I}$ and by doubling the second.

In the special case, $\hat{M}=2 M$, still further simplifications result especially if the relationship

$$
\hat{M}\left(\mathrm{I}-e^{-\hat{M} *}\right)-M\left(\mathrm{x}-e^{-M^{*}}\right)=M\left(\mathrm{I}+e^{-M^{*}}-2 e^{-2^{1-\alpha} M^{*}}\right)
$$

is taken into consideration.

In deciding on a possible change of the retention it might be useful to draw a comparison between the increase in the expected value of the largest claim as derived here and the increase in the total expected loss.

\section{A numerical example}

Beard has given in [4] the empirical claim statistics of an American fire portfolio. If for this investigation we ignore intervals of less than $\$ 50,000$, consider this amount as a unit of account and draw a graph of the remaining values, we get a hyperbolic figure ${ }^{1}$ ). It seemed appropriate, therefore, to assume a Pareto distribution for the basic distribution and, in fact, the $\chi^{2}$ test carried out with the help of the maximum likelihood estimate of $\alpha(\alpha=2,4)$ gave no reason to doubt the hypothesis.

1 The 19 unit intervals between $\$ 50,000$ and $\$$ I million contain respectively the following number of cases: $362 / 82 / 38 / 17 / 10 / 10 / 4 / 4 / 2 / 2 / 3 / 1 /-1-1$ $\mathrm{I} /-/ \mathrm{I} / \mathrm{I}$. Over $\$ \mathrm{I}$ million there were three more claims registered. 
The value calculated for $\alpha$ is relatively low, which shows the "dangerous" character of the distribution. According to the model, claim amounts of \$ I5 million and more must still be expected with a probability of $0.34 \% 0$. Since the three largest claims of the statistics together result (no individual details of these claims are given in the paper) in a claim amount of approximately $\$ 23.5$ million, the curve should certainly not fall off very rapidly. On the other hand, out of the total 54I claims incurred (see the footnote) no fewer than 529 are under the $\$ 0.5$ million mark.

An evaluation of formulas ( 13$)$ and (25) is contained in the following table. It seems reasonable here to proceed on the basis of a retention $M=$ Ioo (i.e. $\$ 5$ million). For computation purposes the tables given in [I2] were used.

TABLE 2

Expected values of the largest claim without and with "truncation"

\begin{tabular}{rrrr}
\multicolumn{1}{c}{$t$} & \multicolumn{1}{c}{$\mu_{H}$} & \multicolumn{1}{c}{$\tilde{\mu}_{H}$} & $\tilde{\mu}_{H} / \mu_{H}$ in $\%$ \\
\hline 100 & 84,5 & 45,5 & 53,8 \\
500 & 266,7 & 83,8 & 31,4 \\
1000 & 437,6 & 94,9 & 21,7 \\
5000 & $138 \mathrm{I}, 3$ & 99,9 & 7,2 \\
10000 & 2266,3 & I00,0 & 4,4 \\
& & $=M$ &
\end{tabular}

If we equate $t$ with the observed number 54I, we get $\mu_{H}=282 . \mathrm{I}$, or upon conversion an amount of about $\$$ I 4 million, a very plausible order of magnitude. With assumed retention of $\$ 5$ million the expected largest claim reduces in the same case to approximately $\$ 4.3$ million.

In the following table a comparison is made between formulas (28) and (29).

TABLE 3

Expected values of the largest and second largest claim without and with "truncation" ( $M=100)$

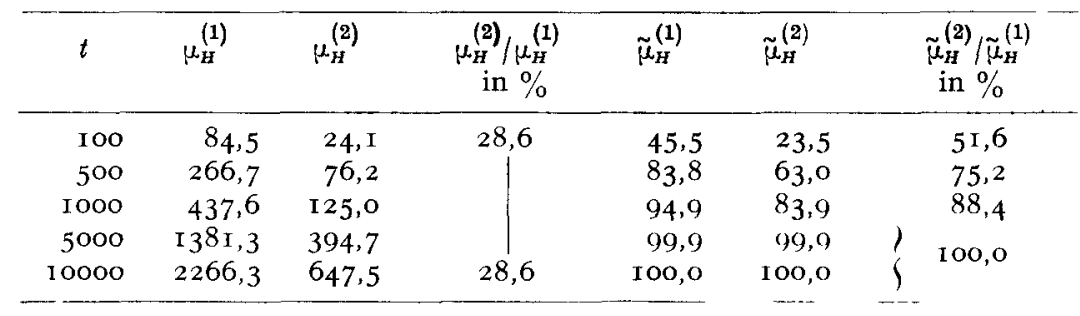


The statements made at the end of section 3 are confirmed here. Another interesting fact is how little, for a small $t$, the expected value of even the second largest claim is affected by truncation.

TABLE 4

Expected values of the largest claim upon a change in the retention

$$
\begin{aligned}
& M=100 \\
& \hat{M}=200
\end{aligned}
$$

\begin{tabular}{rcccccc}
\multicolumn{1}{c}{$t$} & $\tilde{\mu}_{H}^{(1)}$ & $\hat{\tilde{\mu}}_{H}^{(1)}-\tilde{\mu}_{H}^{(1)}$ & $\frac{(2)}{(\mathrm{I})}$ in $\%$ & $\tilde{\mu}_{H}^{(1)}+\tilde{\mu}_{H}^{(2)}$ & $\begin{array}{c}\hat{\tilde{\mu}}_{H}^{(1)}+\hat{\tilde{\mu}}_{H}^{(2)} \\
-\left(\tilde{\mu}_{H}^{(1)}+\tilde{\mu}_{H}^{(2)}\right.\end{array}$ & $\frac{(4)}{(5)}$ in \% \\
\hline & $(1)$ & $(2)$ & $(3)$ & $(4)$ & $(5)$ & $(6)$ \\
\hline 100 & 45,5 & 9,1 & 20,0 & 69,0 & 9,5 & 13,8 \\
500 & 83,8 & 37,5 & 44,7 & 146,8 & 46,2 & 31,5 \\
1000 & 94,9 & 60,3 & 63,5 & 178,8 & 85,2 & 47,7 \\
5000 & 99,9 & 98,4 & 98,5 & 199,8 & 190,9 & 95,5 \\
10000 & 100,0 & 99,9 & 99,9 & 200,0 & 199,6 & 99,8 \\
\hline
\end{tabular}

Finally, the results obtained by application of formulas (30) and (3I) are summarised in the preceding table.

Some of the realisations, which have already been made clear to some extent by the previous explanations, receive confirmation from the numerical values given above.

- If $t$ is large, a doubling of retention practically leads to a doubling of the expected value of the largest claim.

- Taking into consideration also the second largest claim results in a doubling of the amount if $t$ is large, and has little influence if $t$ is small (9.I $\leftrightarrow 9.5)$.

- The percentage increase of the expected values undergoes a pronounced reduction, if $t$ is not much too large, by inclusion of the second largest claim.

\section{REFERENCES}

[I] Ammeter, H., Die Ermittlung der Risikogewinne im Versicherungswesen auf risikotheoretischer Grundlage. Mitteilungen Vereinigung Schweiz. Versicherungsmathematiker Vol. 57 (1957).

[2] Ammeter, H., Note Concerning the Distribution Function of the Total Loss Excluding the Largest Individual Claim. ASTIN Bulletin, Vol. III, Part II, I964.

[3] Ammeter, H., The Rating of "Largest Claim" Reinsurance Covers. Quarterly Letter from the Algemeene Reinsurance Companies, Jubilee Number 2, 1964 . 
[4] BEARD, R. E., Some Statistical Aspects of Non-Life Insurance. Journ. Inst. Act. Students' Society, Vol. XIII, part 3, 1954.

[5] Beard, R. E., Some Notes on the Statistical Theory of Extreme Values. ASTIN Bulletin, Vol. III, Part I, 1963.

[6] Benktander, G., A Note on the Most "Dangerous" and Skewest Class of Distributions. ASTIN Bulletin, Vol. II, Part III, I963.

[7] Benktander, G., and Segerdahl, C.-O., On the Analytical Representation of Claim Distributions with Special Reference to Excess of Loss Reinsurance. Transact. of the XVIth Intern. Congr. of Act., Brussels I960.

[8] Franckx, E., Sur la fonction de distribution du sinistre le plus élevé. ASTIN Bulletin, Vol. II, Part III, I963.

[9] Hofmann, M., Über zusammengesetzte Poisson-Prozesse und ihre Anwendungen in der Unfallversicherung. Mitteilungen Vereinigung Schweiz. Versicherungsmathematiker, Vol. 55 (I955).

[1o] Kupper, J., Wahrscheinlichkeitstheoretische Modelle in der Schadenversicherung Teil II. Blätter der Deutschen Ges. für Versicherungsmathematik, Vol. VI, Part I, r962.

[II] Kupper, J., Some Aspects of Cumulative Risk. ASTIN Bulletin, Vol. III, Part I, I963.

[i2] Pearson, K., Tables of the Incomplete Gamma-Function. Cambridge Univ. Press 1946.

[13] SegerdahI, C.-O., If a Risk Business Goes Bankrupt, when does it occur? A Basis for Fixing Net Retention. Transact. XVth Intern. Congress of Act., New York I957.

[I4] Thépaut, A., Le traité d'excédent du coût moyen relatif (Ecomor). Bull. Trimestriel de l'Inst. des Act. Français, No. 192, I950. 